\title{
Digital Twin for Manufacturing Equipment in Industry 4.0
}

\author{
Tomás Moreno $^{\mathrm{a}}$, António Almeida ${ }^{\mathrm{b}}$, Filipe Ferreira ${ }^{\mathrm{b}}$, Narciso Caldas $^{\mathrm{b}}$, César Toscano $^{\mathrm{b}}$ \\ and Américo Azevedo ${ }^{\mathrm{b}}$ \\ ${ }^{a}$ Faculty of Engineering, University of Porto, Rua Dr. Roberto Frias 378, 4200-465, \\ Portugal \\ b INESC TEC, Rua Dr. Roberto Frias, 4200-465, Portugal
}

\begin{abstract}
The manufacturing industry faces a new revolution, grounded on the intense digitalization of assets and industrial processes and the increasing computation capabilities imposed by the new data-driven digital architectures. This reality has been promoting the Digital Twin (DT) concept and its importance on the industrial companies' business models. However, with these new opportunities, also new threads may rise, mainly related to industrial data protection and sovereignty. Therefore, this research paper will demonstrate the International Data Spaces (IDS) reference model's application to overcome these limitations. Following a pilot study with a Portuguese machine manufacturing company, this paper will demonstrate the development of a cutting and bending machines DT, leveraged on an IDS infrastructure for interoperability, for the plastic and metal industry and its importance to introduce this machine manufacturing company in a new B2B marketplace from the EU project Market 4.0.
\end{abstract}

Keywords. Digital Twin, International Data Spaces, Product life cycle, Industrial Marketplace

\section{Introduction}

As a consequence of the innovative digital technologies inherent to Industry 4.0, markets became more competitive and built towards the customer experience, offering high customization levels [1]. To meet that increasing demand in the customization of services and competitiveness, companies must adapt digital data-driven technologies to provide a flexible response [2] and achieve higher productivity levels [1]. In this digitalization context, data and information are the fundamental aspects of these innovative technologies and the driver [3] for new opportunities and collaborations between industrial companies and services suppliers. Thus, when allowing data exchange between companies, it is possible to develop and implement digital data-driven services [3].

However, when promoting new data-driven services, two major problems arise, mainly related to the massive data generation and, on the other hand, the issues related to data security and protection. These issues stress the development of new mechanisms that enable both machine/data owners and data-services providers to establish data understanding standards and secure data communication protocols and channels. To contribute with research on this domain, the following research questions were defined and proposed: 
RQ1: How can the "Digital Twin" concept be applied at the production systems level to manage and control machines along their life cycle?

RQ2: What new kinds of business models can be created leveraged on the secure and trustable data communication between physical machines and data-driven service providers?

RQ3: How Digital Twin and new secure data communication infrastructures can leverage the introduction of new B2B marketplaces?

The paper has the following structure. Section 2 provides a literature review to provide context and a better understanding of this project's concept and terminology. . Section 3 provides the methodology of the research project in which it will explain the sequence of processes. Section 4 details the general concept and architecture regarding the implementation of the data-driven service, Digital Twin. Section 5 refers to the use case performed with a Portuguese machines manufacturing company within the Market4.0 European project. Finally, in Section 6, conclusions regarding the research project will be drawn based on the case study's state.

\section{Literature Review}

Industry 4.0, as the fourth industrial revolution, deviates from the other industrial revolutions by recurring to the employment of intelligent cyber-physical systems (CPS), Industrial Internet of Things (IIoT), and future-oriented technologies (e.g., Digital Twin) in a manufacturing environment [6-8]. With such technologies, it is possible to take advantage of smart products and production systems to obtain competitive advantages in the highly competitive industrial market [2]. Industries that adopted these innovative technologies started to be referred to as smart factories [9].

\subsection{Digital Twin}

Digital Twin, as the digital representation of the real asset, is formed by three instances, the digital object (1), the physical object (2), and the communication between both objects (3). The digital object is the representation of the physical object in a digital environment and can monitor, emulate, and control the physical behavior. In the Digital Twin, data flows continuously, bidirectional, and in real-time between the objects. DT can be distinguished in two additional stages.

Digital Model (DM) is the digital representation of a non-existing physical object, meaning that the digital object will be the projection of a future physical object [1]. Using a DM before the existence of a physical object, it is possible to emulate the object behavior in a safe (digital) environment. With this, the DM is capable of testing the integration of the physical object in the manufacturing process, reducing the risk of an unsuccessful implementation and the need for physical testing procedures, and optimizing the machine assembly process.

Digital Shadow (DS) differs from the Digital Twin (DT) because it does not support bidirectional communication. In the DS, data flows automatically and in real-time from the physical object to the digital but manual from the digital object to the physical [1]. This real-time nature means that the digital object (in the DT and DS) can monitor and gather data from the manufacturing asset. With this data, it is possible to analyze the asset's performance, and through machine learning, it is possible to recognize patterns 
that had/might lead to a malfunction [1] and schedule maintenance before reaching critical levels of usage.

Regarding the Digital Twin (DT) stage, the digital object can control and act upon the physical object [1] because of the bidirectional communication. Therefore, the DT can schedule and automate high-level tasks [10] to obtain the best performance.

\subsection{International Data Spaces}

With the recent technology progression, smart devices are becoming ever more common and essential to remain competitive in the highly competitive industrial market. However, a vital resource of smart devices is data [3], and with the implementation of such services, data can be unprotected.

International Data Spaces (IDS) initiative aims to create a safe data space where industry enterprises can safely share their data assets [3] with a different enterprise within the IDS ecosystem. IDS is defined by the IDS Association as a "Distributed network of Data Endpoints (i.e., instantiations of the International Data Spaces Connector), allowing a secure exchange of data and guaranteeing Data Sovereignty"[11]. Moreover, IDS allows establishing a secure connection between IDS connectors in which data is shared safely and can only be accessed according to the terms defined by the participants (typically the data owner and data user), guaranteeing data sovereignty for the data owner [11]. Therefore, with IDS, smart devices can be implemented safely, allowing innovative business processes to be created [3].

\section{Methodology}

This study aims to demonstrate how digital twin and the International Data Spaces (IDS) concepts can be used to promote new business models and business opportunities by demonstrating these innovative concept applications in a Portuguese case study, performed within the scope of the Market4.0 European project. To conduct this project, a qualitative research methodology was applied. This research type relies on a smaller data sample to prove an innovative concept [4] going towards this project's goals. Using qualitative research provides a flexible approach that can easily be adapted throughout the case study's development [5]. Initially, an in-depth literature review was performed. In a second phase, it was designed the overall concept of the project. Although the concept presented in this paper has a specific goal, it was designed to be easily adapted in different contexts. Finally, in the last phase of the research, it was followed the implementation of this concept in a Portuguese machine manufacturing company. This phase goal is to gain empirical knowledge to support the conclusions drawn.

\section{Concept model}

Following the industry 4.0 paradigm, innovative data-driven digital services can now be developed based on the Digital Twin technology, leveraged by a secure and reliable communication infrastructure based on IDS technologies. Following this vision, this paper presents a data-driven architecture, combining both the DT, as the digital 
representation of the machine, and the IDS as the trustable and secure communication channel between machine owners and services providers. In concrete, it is demonstrated how this type of architecture may be used to gather machinery data and enable industrial companies to rely on external entities to perform services capable of monitoring, emulate and analyze the machine (Fig. 1).

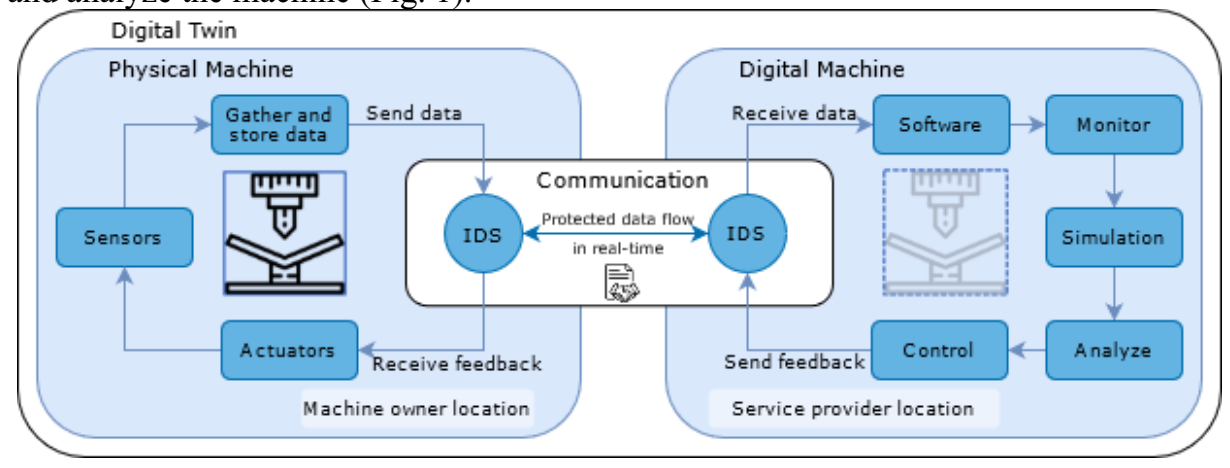

Figure 1. Digital twin implementation concept

As it is possible to see in the image above, on the left side, we have the physical machine, completely digitized through sensors and actuators. On the right side of the image, we have the machine's digital representation processed in the cloud. Here, all the massive data collected is stored and managed following a specific machine's reference data model. All this information can be used to monitor and analyze the machine behavior, taking advantage of artificial intelligence algorithms. As outputs, it is possible to "sell" intelligence about how the machine is performing and what should be done to promote its continued improvement. This bidirectional communication is guaranteed by the IDS channel, where the data owners keep full control of his data.

With the implementation of these value-added services comes the premise of supporting the product life cycle on different product stages (Fig. 2). It is possible to distinguish the concept on two parallel product stages connected through columns: physical product lifecycle (S1) and digital twin lifecycle (S2).

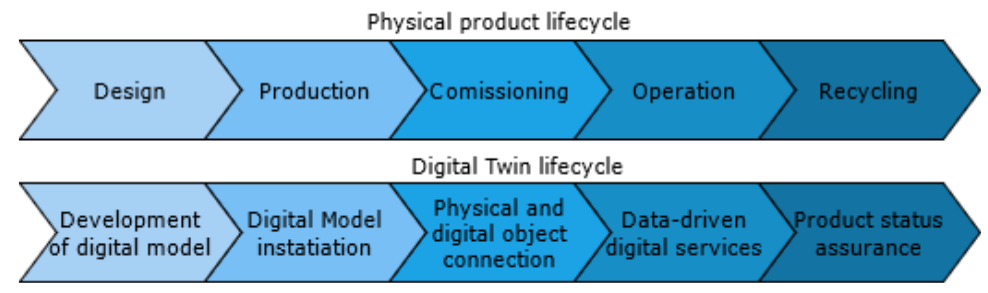

Figure 2. Parallel lifecycles of the product stages

Physical product lifecycle addresses the physical machine processes starting with the machine's design according to the client's specific needs and following the evolution to an operational machine. Digital twin lifecycle follows a Digital Model's conversion from a machine to be built into a Digital Twin that will support the then-built machine's performance.

Overall, with the connectivity between stages, it is possible to support the machine lifecycle in all product stages. As an example, in the first column of processes, it is possible to design a machine according to the clients' need (S1) and convert it into a digital model that will support the machine's implementation (S2). 


\section{GREAT Experience Case Study}

Market4.0 (M4.0) European project aims to create a new B2B industrial marketplace for machines and services that leverages the IDS initiative to ensure data sovereignty between suppliers and customers. This innovative marketplace will support industrial companies from the acquisition to the usage and recycling stage.

The supply of manufacturing equipment is ever so rising, and with this, finding the desired machine is often associated with spending hours on research or direct contact with the manufacturing industries. The M4.0 Equipment Selection Service (ESS) aims to introduce machinery in the Market 4.0 catalog and enable metal and plastic companies to find and configure cutting and bending machines according to their specific needs in a straightforward manner. With this app, the user will be able to specify the desired type of machine, features of the process that will integrate the machine (e.g., minimum/maximum thickness), and material to be processed. After choosing the suitable machine, the user will have the possibility to customize the machine components (e.g., mechanical add-ons, control system) and create a machine configuration that suits it needs. This information will then be presented to the respective vendor in a more effective way, in order to streamline the commercial and industrialization process.

In the M4.0, industrial companies can also find advanced data-driven services for machines performance monitoring and improvement. The Integrated Performance and Maintenance Service (IPMS), composed of a set of innovative data-driven services, will support metal and plastic companies to define conditioned/preventive maintenance plans, leveraged on real-time machine parameters while monitors, calculate, and enhance visibility on machine OEE based on online dashboards. Part of the data-driven services available on this app will be leveraged on the data and parameters collected in real-time from manufacturing machines to a platform that will compile and process the data to generate web-based dashboards and intelligence related to machine usage and performance. Moreover, the IPMS architecture enables monitoring the machines while processing and accessing data generated by them.

The proposed architecture model (Fig. 3) shows the integration between the data provider and the data consumer. In terms of architecture, the left side corresponds to the physical machine. On the right side, it is possible to see the resemblance to the digital machine (Fig. 1), and it corresponds to the service provider location. It includes the Integrated performance and maintenance service (IPMS), responsible for displaying the machine's generated data to the end-user in a graphical interface. All data generated by the machine that needs to be shared with the IDS Data Consumer App is available in an API and communicated/received by an IDS connector.

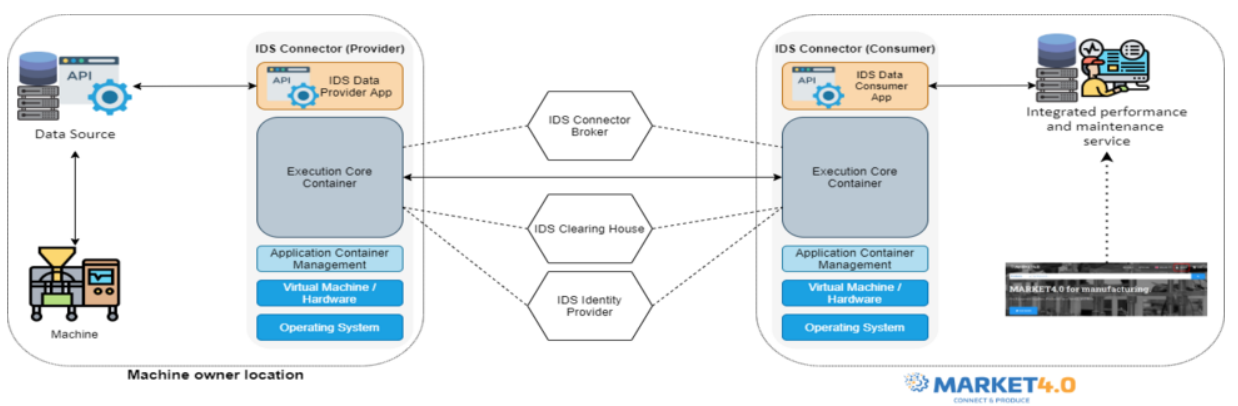

Figure 3. General architecture of the implementation model 


\section{Conclusions}

Based on the GREAT experience previously presented, it was possible to answer the research questions proposed in section 1. Regarding the RQ1, it was demonstrated that the DT can be used to monitor a physical machine and leverage its digital capabilities to monitor and preventive a machine's performance. As stated in Fig. 2, the DT can follow and support the machine lifecycle. The DM can support not only the product customization but also the physical machine implementation and commission with the conversion of the DM into a DT. Addressing the RQ2 demonstrated the importance of IDS to build trustable communication channels between physical machines and datadriven services. With secure data exchange, the machine manufacturer, in addition to selling a machine, can sell and provide data-driven services that will generate additional subscription revenue. Referring to RQ3, the DT will be available and visible through the industrial B2B marketplace, enhancing the selling processes and reaching more potential customers.

\section{Acknowledgements}

This work is financed by National Funds through the Portuguese funding agency, FCT Fundação para a Ciência e a Tecnologia, within project UIDB/50014/2020.

\section{References}

[1] Kritzinger W, Karner M, Traar G, Henjes J, Sihn W. Digital Twin in manufacturing: A categorical literature review and classification. IFAC-PapersOnLine [Internet]. 2018;51(11):1016-22. Available from: https://doi.org/10.1016/j.ifacol.2018.08.474

[2] Aheleroff S, Xu X, Zhong RY, Lu Y. Digital Twin as a Service (DTaaS) in Industry 4.0: An Architecture Reference Model. Adv Eng Informatics [Internet]. 2021;47(October 2020):101225. Available from: https://doi.org/10.1016/j.aei.2020.101225

[3] Data I, Association S. International Data Spaces Ids - a Standard for Data Sovereignty and an. 2019;(September):1-4.

[4] Arabnia HR, Deligiannidis L, Tinetti FG. Frontiers in Education : Computer Science and Computer Engineering. Int Conf Front Educ Comput Sci Comput Eng [Internet]. 2018;13:268. Available from: http://search.proquest.com/openview/837bf2246ca3ee967dd70d72e7d92ce1/1?pqorigsite $=$ gscholar\&cbl $=1976352$

[5] Bhandari P. An introduction to qualitative research [Internet]. 2020 [cited 2021 Apr 9]. Available from: https://www.scribbr.com/methodology/qualitative-research/

[6] Li G, Hou Y, Wu A. Fourth Industrial Revolution: technological drivers, impacts and coping methods. Chinese Geogr Sci. 2017;27(4):626-37.

[7] Sanders A, Elangeswaran C, Wulfsberg J. Industry 4.0 implies lean manufacturing: Research activities in industry 4.0 function as enablers for lean manufacturing. J Ind Eng Manag. 2016;9(3):811-33.

[8] Thames L, Schaefer D. Software-defined Cloud Manufacturing for Industry 4.0. Procedia CIRP [Internet]. 2016;52:12-7. Available from: http://dx.doi.org/10.1016/j.procir.2016.07.041

[9] Hofmann E, Rüsch M. Industry 4.0 and the current status as well as future prospects on logistics. Comput Ind [Internet]. 2017;89:23-34. Available from: http://dx.doi.org/10.1016/j.compind.2017.04.002

[10] Rosen R, Von Wichert G, Lo G, Bettenhausen KD. About the importance of autonomy and digital twins for the future of manufacturing. IFAC-PapersOnLine [Internet]. 2015;28(3):567-72. Available from: http://dx.doi.org/10.1016/j.ifacol.2015.06.141

[11] Otto B, Steinbuß S, Teuscher A, Lohmann S. International Data Space Reference Architecture Model Version 3.0. 2019;(April):1-118. Available from: https:/www.internationaldataspaces.org/wpcontent/uploads/2019/03/IDS-Reference-Architecture-Model-3.0.pdf 